International Journal of Dentistry and Oral Science (IJDOS)

ISSN: 2377-8075

\title{
Cystic Variant of CEOT - A Unique Presentation
}

Saha $\mathrm{A}^{1}$, Sholapurkar $\mathrm{AA}^{2 *}$, Pai $\mathrm{KM}^{3}$, Rao $\mathrm{N}^{4}$

${ }^{1}$ Visiting Consultant, AMRI Hospital, Southern Avenue, Kolkata, India.

${ }^{2}$ Lecturer, Clinical Dentistry and Oral Radiology School of Dentistry, James Cook University, Smithfield, Queensland, Australia.

${ }^{3}$ Professor and Head, Department of Oral Medicine and Radiology, Manipal College of Dental Sciences, Manipal, Karnataka, India.

${ }^{4}$ Professor, Department of Oral \& Maxillofacial Pathology Manipal College of Dental Sciences, Manipal, Karnataka, India.

\begin{abstract}
Calcifying epithelial odontogenic tumor (CEOT) is a rare benign, odontogenic neoplasm. The histologic features of CEOT are unique and serve as the basis for diagnosis. However, among its various histologic subtypes, the cystic variant is extremely rare and less well understood entity, with the MEDLINE search reporting of only two cases till date. We hereby report such unique case and briefly enumerate the differential diagnosis and treatment options. This report illustrates a variant of CEOT that demonstrated ambiguous clinical and radiologic features usually not expected of CEOTs thus making its recognition a challenging task.
\end{abstract}

Keywords: Calcifying Epithelial Odontogenic Tumor; Cystic Variant; Odontogenic Neoplasm; Posterior Mandible.

\section{*Corresponding Author:}

Dr. Amar A Sholapurkar BDS, MDS, FAGE,

Lecturer, Clinical Dentistry and Oral Radiology School of Dentistry, James Cook University, Smithfield, Queensland - 4878, Australia.

Tel: +61455254361

E-mail: dr.amar1979@yahoo.co.in

Received: July 19, 2015

Accepted: August 15, 2015

Published: August 18, 2015

Citation: Saha A, Sholapurkar AA, Pai KM, Rao N (2015) Cystic Variant of CEOT - A Unique Presentation. Int J Dentistry Oral Sci. 2(8), 131-134. doi: http://dx.doi.org/10.19070/2377-8075-1500028

Copyright: Sholapurkar AA ${ }^{\mathcal{O}}$ 2015. This is an open-access article distributed under the terms of the Creative Commons Attribution License, which permits unrestricted use, distribution and reproduction in any medium, provided the original author and source are credited.

\section{Introduction}

Calcifying epithelial odontogenic tumor (CEOT) is a rare, locally aggressive, benign, odontogenic neoplasm accounting for less than $1 \%$ of odontogenic neoplasms [1]. Its clinical and radiographical pictures are not characteristic depending upon the site, size and developmental stages in a range of its composition degeneration. Also its origin and true malignant potential is controversial. Among the histologic features of CEOT, the presence of amorphous amyloid material and liesegang ring calcifications, is unique and serves as the basis for diagnosis [1]. However a true cystic variant of CEOT is extremely rare with only two cases reported till date $[1,2]$. We suggest that the cystic variant though uncommon should be included in the histomorphologic subtyping of this tumor. We report a case of cystic variant of CEOT occurring in the mandible to add to the data in literature and help other clinicians better understand this variation of the classic od-

\section{ontogenic tumor.}

\section{Case Report}

A 29 year old male patient with no premorbid condition presented with a painless swelling over the right posterior region of the lower jaw of four months duration. The swelling occurred insidiously and enlarged gradually. There was no history of pain, tooth mobility or pus discharge in the concerned region.

On examination, a diffuse swelling was noted in the right lower third of the face, measuring approximately $4 \times 4 \mathrm{~cm}$ in size. Intra oral examination (Figure.1) revealed a diffuse swelling obliterating the buccal vestibule in relation to the right mandibular premolarmolar region. The overlying mucosa was erythematous and there was expansion of both the buccal and the lingual cortices in that region. On palpation, the swelling was non-tender, well defined and hard in consistency in the premolar region; however mild fluctuation was felt on the buccal aspect in the molar region which suggested a breach in the buccal cortical plate. There was mobility of the second premolar. Vitality testing with an electric pulp tester exhibited delayed response in all the associated teeth and aspiration from the lesion yielded a yellowish straw colored fluid. Panoramic radiograph (Figure.2) revealed a unilocular radiolucency (with no evidence of calcifications) in the right body of the mandible extending anteroposteriorly from the distal of canine to the mesial root of third molar. Small, thin, opaque trabeculae were seen crossing the radiolucency in many directions. The radiolucency exhibited scalloped borders and extended between the roots of the first and second premolar thereby significantly altering their axial inclination. The inferior alveolar canal was displaced inferiorly and the roots of both the premolars showed evidence of diffuse external resorption. A CT scan study (Figure. 3) of the mandible showed the expansion of the buccal and lingual cortical plates (arrows indicating the site of perforation) along with perforation of the buccal cortex in the premolar region and 
Figure 1. The swelling in the right mandibular alveolar process in the premolar-molar region.

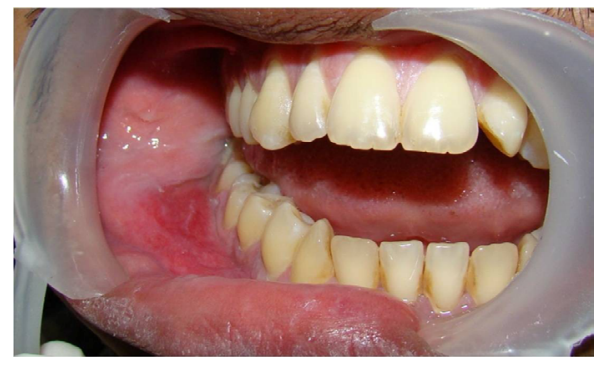

Figure 2. Cropped panoramic image showing multilocular radiolucency in the right body of the mandible extending from the distal of canine to the third molar.

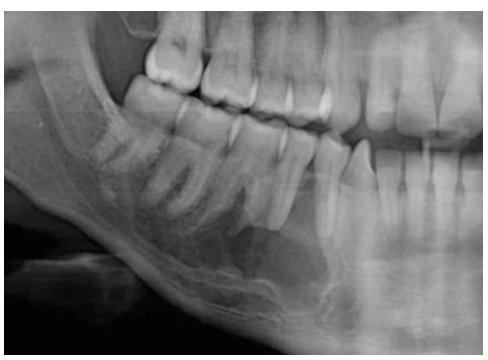

Figure 3. A CT scan study of the mandible confirming the expansion of the buccal and lingual cortical plates along with perforation of the buccal cortex (red arrow) in the premolar region and the lingual cortex (white arrow) in the molar region.

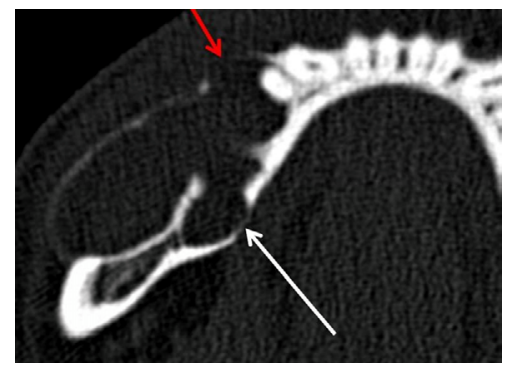

the lingual cortex in the molar region.

Based on the history, clinical examination and imaging findings, our clinical differential diagnosis included Solid-multicystic ameloblastoma, Calcifying cystic odontogenic tumor (CCOT), and Calcifying epithelial odontogenic tumor. Considering the age of our patient, the location of the lesion and its clinical presentation, ameloblastoma, which is the most common odontogenic neoplasm of the jaws was considered to be the most likely possibility. Solid-multicystic ameloblastomas account for $92 \%$ of all intraosseous amelobastomas [3]. They occur in patients mainly in their third to fifth decades and majority arise in the mandible $(85 \%)$, most commonly in the molar and ramus regions. Small tumors are usually incidental findings on routine radiographs, and larger lesions produce local symptoms of pain, swelling, malocclusion, and paresthesia [4]. Radiographically, solid ameloblastomas appear as multilocular or "soap-bubble" radiolucencies. Although the imaging studies in our case revealed an unilocular lesion, solid ameloblastoma was considered in the differential diagnosis because evidence from CT studies have shown that the term multicystic may be a misnomer in many conventional ameloblastomas [5]. The radiographic spaces show scalloping resorption of the delimiting cortical plates, giving the illusion of multicystic spaces rather than truly septated compartments. Impacted teeth are associated with $15 \%-40 \%$ of all cases [5]. Microscopically, solid ameloblastomas may frequently undergo secondary cystic change and this may be the cause for the cyst-like radiographic appearance in our case and the presence of a straw colored aspirate.
Although, the calcifying cystic odontogenic tumor (CCOT), also known as calcifying odontogenic cyst (COC) is an uncommon lesion it was included in our differential diagnosis because of its strong clinical and radiological resemblance with our case. CCOT occurs most commonly in the second and third decades of life and affects both genders with almost equal frequency. The mandible is most commonly affected with a greater frequency of cases occurring in the premolar-molar region. The cyst clinically presents as a painless, expansile lesion and is often found in association with an odontoma or impacted tooth. Radiographically, CCOT presents as unilocular, well defined radiolucency or radiolucency with foci of opacification.

Calcifying epithelial odontogenic tumor (CEOT) was also considered but somewhat lower down the order in the differential diagnosis because of the following reasons. Firstly, its relatively weak correlation of the age (CEOT's are more common in $4^{\text {th }}$ to $6^{\text {th }}$ decades of life) and absence of its association with an unerupted/ impacted tooth were two factors against our case, but not major factors to exclude it from the differential diagnosis. Secondly, radiographic findings usually reveal well defined, unilocular / multilocular radiolucencies with calcifications [6] (classically described as resembling 'wind driven snow'). However, calcifications were not detected in our case.

To determine the true nature of the lesion, an incisional biopsy was performed under local anaesthesia. The tissue obtained was 
submitted for histopathological examination which revealed the presence of cystic lumen lined by odontogenic epithelium, large areas of amorphous amyloid material and comparatively fewer amount of liesegang ring calcifications (Figure 4A, B and C). These findings prompted a final diagnosis of cystic variant of calcifying epithelial odontogenic tumor. In view of the diagnosis, the patient was advised surgical excision of the lesion and during the surgical procedure both the premolars and the first molar were extracted. The patient has been under continuous surveillance and has not exhibited any evidence of recurrence till date.

\section{Discussion}

The classic Calcifying epithelial odontogenic tumor (CEOT) is classified as an uncommon, benign, odontogenic neoplasm that is exclusively epithelial in its tissue of origin. It was first recognized as a separate entity by Dr. Jens J. Pindborg in 1958, justifying its alternative name of Pindborg's tumor [1]. To the best of our knowledge only less than two hundred such types of cases have been reported in the English literature till date [1, 7]. However we are aware of only two cases of the cystic variant of CEOT in the English literature reported by Rajaram Gopalkrishnan et al [2] in 2006 and Channappa NK et al in 2012 [1]. In both the documented cases the cystic lining showed evidence of CEOT similar to our case. The cystic variant of CEOT may result from a cystic degeneration of a solid tumor or may exhibit a cystic pattern of growth similar to that encountered frequently in Unicystic ameloblastomas or it may originate from the neoplastic transformation of dental follicular lining epithelium of an impacted tooth or epithelial lining of a dentigerous cyst.

Classically, the tumor presents as a slow-growing, painless, bony hard swelling causing cortical bone expansion. Most of the CEOT's occur in the posterior mandible [8] and are frequently associated with an unerupted tooth. Rare extraosseous examples have also been reported with the anterior gingiva being the most frequent location [9]. Radiographically, CEOTs appear as unilocular or multilocular radiolucencies with or without internal radiopacities. In one study of 67 Pindborg tumors, [6] the mixed radiolucent and radiopaque pattern occurred most often $(65 \%)$, followed by the completely radiolucent pattern $(32 \%)$ and, least often, the totally radiopaque pattern (3\%). Radiopacities present due to calcifications in the tumor are classically described as resembling 'wind driven snow' and is the distinguishing radiographic feature of CEOT. The radiographic presentation of the two reported cases [1, 2] revealed unilocular radiolucency with impacted tooth and irregular calcifications in close association with it. However, our case was very different and unique radiographically where the lesion was not associated with an impacted tooth and there was no evidence of calcification. Microscopically, the tumor typically shows the presence of discrete islands, strands or sheets of polyhedral epithelial cells in a fibrous stroma, large areas of amorphous amyloid like extracellular material, and liesegang ring calcifications within the amyloid material. Other variants of the classic appearance have been recognized and include tumors with: (a) minimal amyloid-like material and calcification, (b) prominent amyloid- like material and minimal epithelial cells, and (c) a predominantly clear-cell variant of CEOT [10]. CEOTs displaying true malignant features have also been reported [11].

CEOTs are less aggressive tumors [12] as compared to ameloblastomas and hence, conservative tumor excision is the treatment of choice as was done for our patient. Although CEOT has been known to have a recurrence rate of $14 \%$ [10], our patient's lesion did not show any signs of recurrence during the follow up period similar to the first documented case of cystic CEOT. However the prognosis of this variant can be better estimated after follow up of more such cases are reported.

Figure 4A. Area stained with H\&E showing Cystic lumen lined by odontogenic epithelium (10X original magnification).

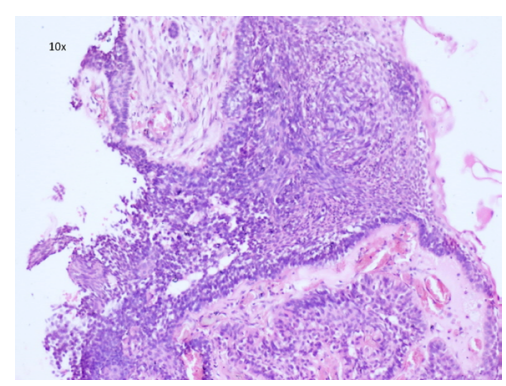

Figure 4B. Area stained with H\&E showing amorphous amyloid material (100X original magnification).

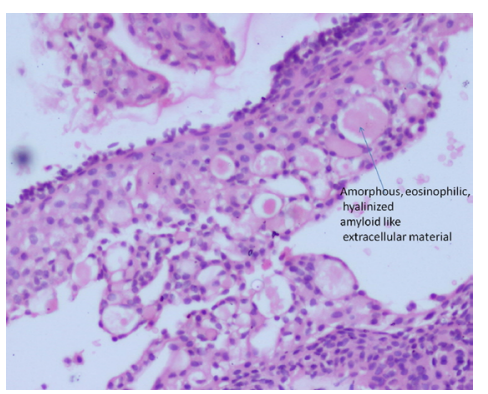


Figure 4C. Area stained with H\&E showing liesegang ring calcifications (green arrows).

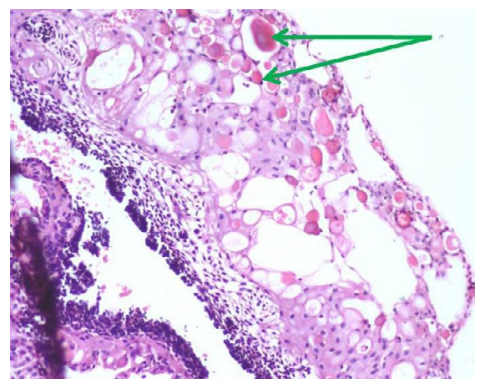

\section{References}

[1]. Channappa NK, Krishnapillai R, Rao JB (2012) Cystic variant of calcifying epithelial odontogenic tumor. J Investig Clin Dent 3(2): 152-156.

[2]. Gopalakrishnan R, Simonton S, Rohrer MD, Koutlas IG (2006) Cystic variant of calcifying epithelial odontogenic tumor. Oral Surg Oral Med Oral Pathol Oral Radiol Endod 102(6): 773-777.

[3]. Reichart PA, Philipsen HP, Sonner S (1995) Ameloblastoma: biological profile of 3677 cases. Eur J Cancer B Oral Oncol 31B(2): 86-99.

[4]. Black CC, Addante RR, Mohila CA (2010) Intraosseous ameloblastoma. Oral Surg Oral Med Oral Pathol Oral Radiol Endod 110(5): 585-592.

[5]. Kessler HP (2004) Intraosseous ameloblastoma. Oral Maxillofac Surg Clin North Am 16(3): 309-322.

[6]. Kaplan I, Buchner A, Calderon S, Kaffe I (2001) Radiological and clinical features of calcifying epithelial odontogenic tumor. Dentomaxillofac Radiol 30(1): 22-28.

[7]. Deboni MCZ, Naclério-Homem MDG, Pinto Jr DS, Traina AA, Cavalcanti
MGP (2006) Clinical, Radiological and Histological Features of Calcifying Epithelial Odontogenic Tumor: Case Report. Braz Dent J 17(2): 171-174.

[8]. Makos CP, Nikolaidou AJ (2004) Calcifying epithelial odontogenic tumour or Pindborg's tumour: features and behaviour in relation to a case. Int Dental J 54(6): 457-460.

[9]. Houston GD, Fowler CB (1997) Extraosseous calcifying epithelial odontogenic tumor: report of two cases and review of the literature. Oral Surg Oral Med Oral Pathol Oral Radiol Endod 83(5): 577-583.

[10]. Anavi Y, Kaplan I, Citir M, Calderon S (2003) Clear-cell variant of calcifying epithelial odontogenic tumor: clinical and radiographic characteristics. Oral Surg Oral Med Oral Pathol Oral Radiol Endod 95(3): 332-339.

[11]. Cheng YS, Wright JM, Walstad WR, Finn MD (2002) Calcifying epithelial odontogenic tumor showing microscopic features of potential malignant behavior. Oral Surg Oral Med Oral Pathol Oral Radiol Endod 93(3): 287-295.

[12]. Philipsen HP, Reichart PA (2000) Calcifying epithelial odontogenic tumour: biological profile based on 181 cases from the literature. Oral Oncol 36(1): $17-26$. 